\title{
Eclipsing Binary Stars in Nearby Galaxies
}

\author{
Ian Todd • Don Pollacco • Ian Skillen • D. M. Bramich • \\ Steve Bell · Thomas Augusteijn
}

Received: 1 November 2005 / Accepted: 1 February 2006

(C) Springer Science + Business Media B.V. 2006

\begin{abstract}
We briefly discuss the survey programme we are conducting to detect eclipsing binaries in local group galaxies. Some lightcurves from studies of M31, IC 1613 and NGC 6822 are presented along with details of future work.
\end{abstract}

Keywords Stars · Local group · Eclipsing binaries · Distance scale

\section{Introduction}

Local Group Galaxies serve as a natural laboratory for the study of astrophysical phenomenon over a wide range of environments. The determination of their distance allows the calibration of standard candles such as Cepheids, enabling the distance scale to be accurately extended beyond the Local Group. Ultimately, one goal is to provide a better determina-

I. Todd $(\bowtie) \cdot$ D. Pollacco

Dept of Physics and Astronomy, Queen's University Belfast, Antrim, BT7 1NN, UK

e-mail: i.todd@qub.ac.uk

I. Skillen

Isaac Newton Group of Telescopes, Apartado 321, E-38700 Santa

Cruz de la Palma, Tenerife, Spain

D. M. Bramich

Dept. of Physics and Astronomy, University of St. Andrews, Fife, KY16 9SS, UK

S. Bell

HM Nautical Almanac Office, CCLRC Rutherford Appleton

Laboratory, Oxon, OX11 0QX, UK

T. Augusteijn

Nordic Optical Telescope, Apartado 474, E-38700 Santa Cruz de

la Palma, Tenerife, Spain tion of the Hubble Constant, which is known to a precision of around 10\% (Altavilla et al., 2004). There are two major factors that contribute to this uncertainty: the absolute distance to the Large Magellanic Cloud and the possible dependance of the Cepheid Period-Luminosity relationship on metallicity.

Eclipsing Binaries (EBs) however allow the possibility of distance determination to better than $5 \%$, as it is possible to determine the absolute properties of the components with great accuracy (Andersen, 1991; Clausen, 2004). Traditionally, detached eclipsing binaries are thought to be more appropriate for distance determination, but even semi-detached eclipsing binary systems can be used, as they contain more information throughout their lightcurves and are subject to more constraints (Wilson, 2004). Modern software can fit these eclipsing binary lightcurves using the latest physical models.

The use of eclipsing binaries for distance determination is not a new concept. Gaspshkin (1968) first suggested their use in the Magellanic clouds. The distance to the SMC has also been derived using eclipsing binaries (Hilditch et al., 2005), and also Bonanos et al. (2006) report on the first distance to M33 based on an eclipsing binary.

\section{Data reduction}

The data were reduced using the difference imaging software of Bramich et al. (2005). This method has several advantages over aperture photometry and profile fitting in crowded extragalatic fields. Difference Image Analysis (DIA) matches the point-spread-function (PSF) between frames in a time series by generating a best-seeing reference frame and then degrading that frame to the seeing of the other frames via the generation of a kernel solution. The kernel solution models 
Fig. 1 A selection of eclipsing binary lightcurves from M31
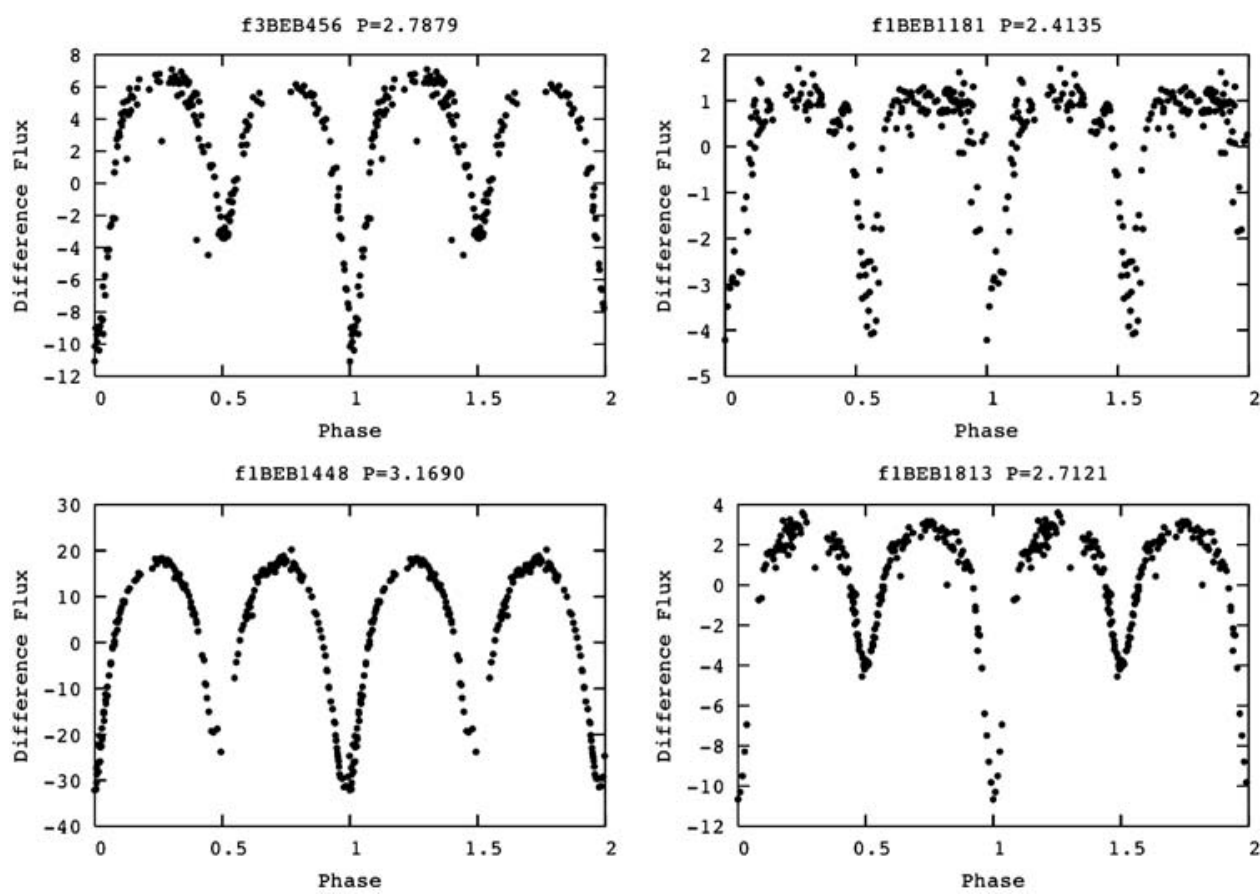

the changes in the PSF from frame-to-frame as well as within a frame. In this way, a difference flux lightcurve is generated for each star found on the reference, and converted to magnitudes by measuring the brightness of each of the stars on the reference. In crowded fields however, problems with blending may cause star magnitudes to be measured inaccurately, resulting in erronous amplitudes of lightcurves. Thus, it is better to clearly state that this will be the case or give lightcurves in units of differential flux. A more detailed account of these issues can be found in Todd et al. (2005).

\section{Observations and results}

The data for M31 were extracted from the Isaac Newton Group Data Archive ${ }^{1}$ over a four-year baseline. All relevant data over this period were extracted from the archive. ${ }^{2}$ The Wide Field Camera (WFC) attached to the Issac Newton Telescope (INT) on La Palma was used to collect all M31 data. Data for IC 1613 and NGC 6822 were collected using MOSCA on the Nordic Optical Telescope (NOT).

\subsection{M31}

M31 is an important Local Group Galaxy for stellar population studies and distance determination. Its proximity to

\footnotetext{
${ }^{1}$ ING Archive at http://archive.ast.cam.ac.uk.

${ }^{2}$ see Acknowledgements.
}

the Galaxy means that its bright stellar population can be resolved relatively easily. It has been shown to have many eclipsing binaries and Cepheids. As it is a spiral galaxy, geometric effects are relatively easy to model (unlike the irregular LMC and SMC), and it is the first step of the TullyFisher relation for spiral galaxies. It is therefore important that its distance is known accurately and systematics are identified.

We have used DIA to extract lightcurves for variable objects in the Eastern Spiral Arm of M31 over a four year baseline. Using a matched filter approach to detect binaries and Phase Dispersion Minimization (PDM) to determine periods (Stellingwerf, 1978), we have discovered around 280 binary systems in our data, with 98 newly discovered systems (Todd et al., 2005) and 128 have well determined periods. The data also contains many Cepheids, another primary distance indicator. A selection of binary lightcurves is shown in Fig. 1.

\subsection{IC 1613 and NGC 6822}

We have also been surveying IC 1613 and NGC 6822 for variability. Previously, Baade surveyed IC 1613 for variable content (Sandage, 1971). Antonello (2002) have discovered at least nine eclipsing binaries in IC 1613 and NGC 6822. So far, we have discovered around one hundred eclipsing binary lightcurves in these galaxies and are currently collecting more data to increase our sampling. We expect to have a catalogue of bright eclipsing binaries in these galaxies in the future. 


\section{Other work}

Currently we are conducting surveys in other galaxies which are mostly beyond the Local Group. It is important that in the study of eclipsing binaries and particularly in the case of Cepheids, we survey environments with different metallicities to investigate its effect on the Period-Luminosity relationship. Galaxies that are currently under investigation include NGC 2403, M81, M83, Leo A, NGC 3109 and Sex A. We intend to characterise the Cepheid population of these targets.

Acknowledgements We would like to thank the ING Data Archive at Cambridge for maintaining and making accessible the data that made this study possible, and the multitude of observers and their associates who took time at the telescope through the various surveys to collect this data on the INT (T. Augusteijn, P. Boyce, M. Bremer, P. Bunclark, R. Corrandi, P. Dobbie, A. Ferguson, S. Hodgkin, C. Jordi, Kenynon, P. Lacerda. J. Mendez, S. Rawlings, V. Reyes, I. Ribas, S. Rix, S. Smartt,
W. Sutherland, P. Sorenson, N. Walton). We would also like to thank the Nordic Optical Telescope for allowing our group time to make our own observations of IC 1613 and NGC 6822.

\section{References}

Altavilla, G., Fiorentino, G., Marconi, M., Musella, I., Cappellaro, E., Barbon, R. et al.: MNRAS 349, 1344 (2004)

Andersen, J.: A\&AR 3, 91 (1991)

Antonello, E., Fugazza, D., Mantegazza, L., Stefanon, M., Covino, S.: A\&A 386, 860 (2002)

Clausen, J.V.: NewAR 48, 679 (2004)

Bonanos, A.Z., Stanek, K.Z., Kudritzki, R.P. et al.: DOI: 10.1007/ s10509-006-9112-1 (2006)

Bramich, D.M. et al.: MNRAS 359, 1096 (2005)

Gasposhkin, S.: PASP 80, 556 (1968)

Hilditch, R.W., Howarth, I.D., Harries, T.J.: MNRAS 357, 304 (2005)

Sandage, A.: ApJ 166, 13 (1971)

Stellingwerf, R.F.: ApJ 224, 953 (1978)

Todd, I., Pollacco, D., Skillen, I., Bramich, D.M., Bell S.A., Augusteijn, T.: MNRAS 362, 1006 (2005)

Wilson, R.E.: NewAR 48, 695 (2004) 